\title{
A Study on Distribution of Various Morphological Pulmonary Mass Lesions Using Computed Tomography Guided Fine Needle Aspiration Cytology
}

\author{
${ }^{1}$ Dr.U.Parameswari Babu, ${ }^{2}$ Dr.B.V.Sai Prasad, ${ }^{3}$ Dr.D.Ranga Rao ${ }^{4}$ NHanumanth \\ ${ }^{I}$ (Assistant Professor, Department Of Pathology, Dr.PSIMS \& RF, Chinnaoutpalli, India) \\ ${ }^{2}$ (Associate Professor, Department Of Pathology, S.V.Medical College, Tirupati, India) \\ ${ }_{3}^{3}$ (Professor \& HOD, Department Of Pathology, Dr.PSIMS\& RF, Chinnaoutpalli, India) \\ ${ }^{4}$ (Lecturer in statistics, Department of community medicine, Dr.PSIMS\&RF, Chinnaoutpalli,India)
}

\begin{abstract}
:
Background: CT guided Fine Needle Aspiration Cytology (FNAC) isthe first investigation of choice for intra thoracic mass lesions. It is very easy, safe, and provides real time visual access for aspiration of deeper tissues even though few millimeters in size. Objective: Present study was to evaluate the various spectrums of morphological mass lesions in the lung and determine effectiveness of CT guided FNAC.Materials and methods: This was a descriptive, cross sectional study on 50 patients, over a period of 1 year from April 2015 to March 2016, who underwent CT guided aspirationand their diagnoses were confirmed by appropriate methods.The results were analyzed and interpreted statistically.The study was approved by the Institutional Ethics Committee.

Results: Out of 50 cases, $76 \%$ ( $n=38$ ) were males while rest of were females $24 \%(n=12)$. For interpretation of specific diagnosis material was obtained $84 \%(n=42)$ of cases. Most of thelung lesions were malignant $76 \%($ $n=38$ ) with in this squamous cell carcinoma56\% ( $n=28)$ is most common, followed by adenocarcinoma $12 \%$ $(n=6)$ and inflammatory granulomatous $8 \%(n=4)$ lesions.Conclusions: CT image guided Fine needle aspiration cytology provide more accurate, a non-invasive technique and extremely valuable in diagnosis of pulmonary mass lesions with minimal post aspiration complications.
\end{abstract}

Keywords: Computed tomography guided fine needle aspiration cytology, Lung mass lesion

\section{Introduction}

The Idea behind Fine needle aspiration cytology (FNAC) was, the needle introduce in to abnormal tissue and obtain fragmentsof tissue through it. Though it was started in $19^{\text {th }}$ century many pioneers in this work developed needles and aspiration guidelines togetherwith technological advances for best cell yielding techniques in aspiration cytology withexfoliative cytologyalso ${ }^{1}$. Following success in this area and more focused of interest on preoperative preliminary diagnosis of all types of masses not only neoplastic but also valuable in inflammatory and degenerative disorders, in addition to further used for microbiological and biochemical study of tissue samples ${ }^{2,3}$. However image guided aspiration was the most efficient method for community based practice to the advantage of patient in term of convenience. CT guided FNAC is safer, easy, inexpensive and provides real time visual access for mass lesions in the intra-thoracic, retroperitonium, skull base and also helps in cross sectional localization of needle tipwithin a lesion, very accurately . It also provides, follow up the post aspirational complications like hematoma and pneumothorax. The main purpose of this study is to evaluate the spectrum of various morphological mass lesions in the lung and determine the accuracy of CT for assisted FNAC in diagnosis of pulmonary mass lesions.

\section{Materials and methods}

This was a cross sectional institutional based study was undertaken , 50 patients who were underwent CT guided trans-thoracic FNAC of mass lesions in lung, over a period of 1 year from April 2015 to 2016 referred to department of pathology, Dr.PSIMS\& RF, Chinoutapalli. The inclusion criteria of this study were patients with clinically suspected mass lesions in the lung. Detailed clinical data including personal history of smoking, prior contact of pulmonary tuberculosis etc.Was obtained. FNAC performed with 20 or 22 gauge disposable lumbar puncture needle after obtaining proper informed consent. Arterio-venous malformations, bleeding diathesis and pulmonary hypertension were ruled out. Premedication of mild generalized anesthesia was given to the elderly patients. The smears were stained with hematoxylin and eosin, following alcohol fixation. The patient was followed for 2 hours; repeat CT was done and keep tracking of any post aspirational complications. The study was approved by the ethical committee of the institution. 


\section{Results}

Total number of 50 patients were selected for this study,wasshowed male preponderance with 38 were males $(76 \%)$ and rest 12 were females(24\%). The age ranged from widely 20years to 80 years. The Male and female ratio is 3.1:1. The youngest patient was 23 year old male and cytologicallydiagnosed as pulmonary Koch's. The significant finding in this present study was that 20 out of 38 male patients, presentedat the age group of 51-60 years and only 5 female cases were present in this age.

Pulmonary parenchymal mass lesions, 62\% $(n=31)$ were found in the right lung and rest of the $38 \%$ $(n=19)$ located in left, and most of those located in the upper zone followed by peripheral lesions.

Out of 50 cases, definitive cytological diagnosis made out $84 \%(n=42)$ of cases and rest of $16 \%(n=8)$ cases were inconclusive and descriptive report was given to the patient.

Most of the cytological diagnosed cases were malignant $76 \%(n=38)$ and $8 \%(n=4)$ of cases were diagnosed as granulomatous inflammation.

In the present study most of pulmonary mass lesions, were malignant within these Squamous cell carcinoma $56 \% \quad(n=28)$ was most common malignant tumor,followed by adenocarcinoma $12 \%$ $(n=6)$.Microscopically both squamous and adenocarcinoma showed classical cyto-morphological features.All most all the cases associated with active smoking.

All the squamous cell carcinoma 56\% (28) cases diagnosed in our study are located in the central and peripheral mass lesions. In case of adenocarcinoma $12 \%(n=6)$ most of were located in the periphery.

Other tumorswere $6 \%(n=4)$ in this study,4\% $(n=4)$ were mesotheliomas, $2 \%(n=2)$ lymphoma and metastatic each respectively.

\section{Discussion}

The present study was carried out to categorize the various intra-thoracic lesions who underwent CT guided FNAC. Specific diagnosis obtained $84 \%$ of cases, which was comparable to other studies like Arslan et ${ }^{4}$ al is $88 \%$. In this present study average ageranges varies widely from third to eighth decade, showing the male preponderance with a male - female ratio 3.1:1. Peak age incidence (51-60) was same as that as shah S et al ${ }^{5}$. The mean age (56.8 years) was also almost similar ( 56.4 years) to a study conducted by Singh $e a^{6}{ }^{6}$. However, Wallace $e t$ al.$^{7}$ showed a slightly higher mean age of 61.3 years.

Males $(76 \%)$ were showing significance preponderance in our present study. This is almost similar in saha et al ${ }^{8}$. In recent studies also showed males were little higher than in our study i.e. $88 \%{ }^{5}$ and $80.6 \%{ }^{9}$ However other studies by sing et $\mathrm{al}^{6}$ andWallace et $\mathrm{al}^{7}$ showed significantly lower incidence of male patients $52 \%$ and $55.7 \%$ respectively.

Definitive cytological diagnosis was made out in 42 out of 50 cases (84\%) with diagnostic accuracy of $84 \%$ with high incidence of malignancy $(76 \%)$ also found in similar other studies ${ }^{6,9,10,11,12}$. Among 42 cases of pulmonary mass lesion, most common $56 \%(\mathrm{n}=26)$ cases were squamous cell carcinoma followed by adenocarcinoma $12 \%$ and inflammatory granulomatous lesions were $16 \%$. This order of frequency was also found in other national studies $5,9,13,14$. Tumor frequency order was reversed asadenocarcinoma was reported to be significantly higher than that of squamous cell carcinoma in recent studies by Tan et al., ${ }^{15}$ and Madan et al. ${ }^{16}$

Other $16 \%(n=4)$ pulmonary mass lesions, $4 \%(n=2)$ were mesothelioma, $2 \%(n=1)$ were lymphoma and metastatic tumor each .metastatic tumor was found where primary was known case of prostatic carcinoma.

Both the benign and non-neoplastic lesions $8 \%(n=4)$ were diagnosed as granulomatous inflammation consistent with koch's. The incidence of inconclusive cases were $16 \%(\mathrm{n}=8)$ in the present study this is comparable with other studies sing et al ${ }^{6}$. Inconclusive results were due to scanty aspirate with low cellularity and tissue mixed with hemorrhage.

In our study, CT guided FNAC showed almost perfect correlation with histological diagnosis in comparison to radiological opinion. Guided FNAC was found to be highly accurate in diagnosis of lung mass as almost similar shown by previous studies. ${ }^{13,16}$ so, CT guided FNAC alone provide definitive diagnosis and with confidence for further selection of treatment modalities and to avoid unwanted surgeries in patients with lung mass lesions.

One of the interesting finding in our present study was more than $70 \%$ patients were active smokers, showing strong association with development of malignancy.In 1964, the US Public Health Service published a landmark report from the Surgeon General on smoking and its effects on health. seminal report stated that Cigarette smoking was causally related to lung cancer in men $^{17}$.

\section{Conclusions}

CT guided FNAC of intra-thoracic mass lesions is very easy, inexpensive, safe, and high diagnostic accurate procedure, with minimal post aspirational complications. It provide accurate diagnosis and cytomorphological sub classification of various lung lesions. Inflammatory lesions like granulomatous diseases, tuberculosis also can be diagnosed with certainty.It also avoids unnecessary thoracotomy for diagnostic 
purposes. Our present studyevaluated, various mass lesion in the lung and determines diagnostic accuracy of CT guided needle aspiration of pulmonary mass lesions, and the results were mostly comparable with those of other national and international series of studies. The definitive diagnosis obtained by CT guided FNAC, which helps in further selection of effective treatment modalities.

\section{References}

[1]. Söderström N. Thin needle aspirationbiopsy. Letter.ActaCytol 1980;24:468

[2]. Bedrossian CWM. Bridging the gap between cytopathology and surgical pathology.DiagnCytopathol 1995;12:1-2

[3]. Strigle SM, Rarick MU, Cosgrove MM, et al. A review of the fine-needle aspiration cytology findings in human immunodeficiency virus infection.DiagnCytopathol 1992;8:41-52

[4]. Arslan S, Yilmaz A, Bayramgurller B, Uzman O, Unver E, Akkaya E. CT guided fine needle aspiration of pulmonary lesions: Accuracy and complications in 294 patients. Med SciMonit. 2002;8

[5]. Shah S, Shukla K, Patel P. Role of fine needle aspiration cytology in diagnosis of lung tumors - A study of 100 cases. Indian J PatholMicrobiol 2007;50:56-8.

[6]. Sing JP. Garg L, Setia V. Compared tomography (CT) guided transthoracic needle aspiration cytology in difficult thoracic mass lesions - not approachable by USG. Indian J Radiol Imaging 2004;14:395-400

[7]. Wallace MJ, Krishnamurthy S, Broemeling LD, Gupta S, Ahrar K, Morello FA Jr, et al . CT-guided percutaneous fine-needle aspiration biopsy of small $(\leq 1-\mathrm{cm})$ pulmonary lesions. Radiology 2002;225:823-8

[8]. sahaA,KumarK,ChoudhauriMK.Computed tomography-guided fine needle aspiration cytology of thoracic mass lesions: A study of 50 cases. J Cytol 2009;26:55-9

[9]. Bandyopadhyay A, Laha R, Das TK, Sen S, Mangal S, Mitra PK. CT guided fine needle aspiration cytology of thoracic mass lesions: A prospective study of immediate cytological evaluation. Indian J PatholMicrobiol 2007;50:51-5.

[10]. Stewart CJ, Stewart IS. Immediate assessment of fine needle aspiration cytology of lung. J ClinPathol 1996;49:839-43.

[11]. vanSonnenberg E, Casola G, Ho M., Neff CC, Varney RR, Wittich GR, et al. Difficult thoracic lesions: CT-guided biopsy experience in 150 cases. Radiology 1988;167:457-61

[12]. Abrari A, Aziz M, Haq R. Cytology of lung tumors - study of 60 cases with emphasis on accuracy and problem areas. J Cytol 2003;20:79-81

[13]. Jaya Shankar E, Pavani B, Chandra E, Reddy R, Srinivas M, Ashwin Shah. Computed tomography guided percutaneous thoracic: Fine needle aspiration cytology in lung and mediastinum. J CytolHistol. 2010;107:1-3.

[14]. Basnet SB, Thapa GB, Shahi R, Shrestha M, Panth R. Computed tomography guided percutaneous transthoracic fine needle aspiration cytology in chest masses. JNMA J Nepal Med Assoc. 2008;47:123-7

[15]. Tan KB, Thamboo TP, Wang SC, Nilsson B, Rajwanshi A, Salto-Tellez M. Audit of transthoracic fine needle aspiration of the lung: Cytological sub classification of bronchogenic carcinomas and diagnosis of tuberculosis.Singapore Med J. 2002;43:570-5.

[16]. Madan M, Bannur Evaluation of FNAC in lung diseases. Turk J Pathol. 2010;26:1-6.

[17]. U.S. Public Health Service, office of the Surgeon General: The health consequences of smoking.National Clearinghouse for Smoking Health. 1972

Figure no.1: Photomicrograph showing features of Squamous cell carcinoma of lung (H\&E stain x10 magnification (inset shows large polygonal hyper chromatic cells)

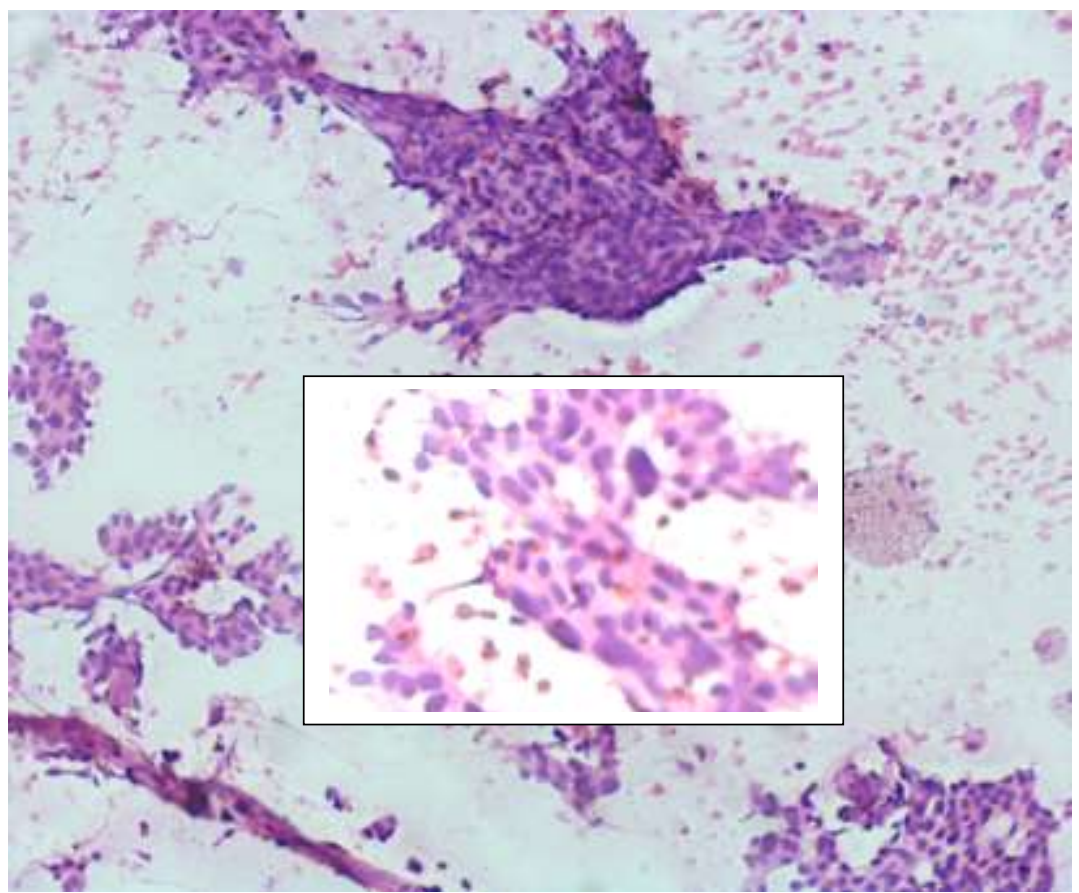


A Study On Distribution Of Various Morphological Pulmonary Mass Lesions Using Computed...

Figure no.2: Photo micrograph showing features of Adenocarcinoma of lung (H\&E stain x10 magnification)

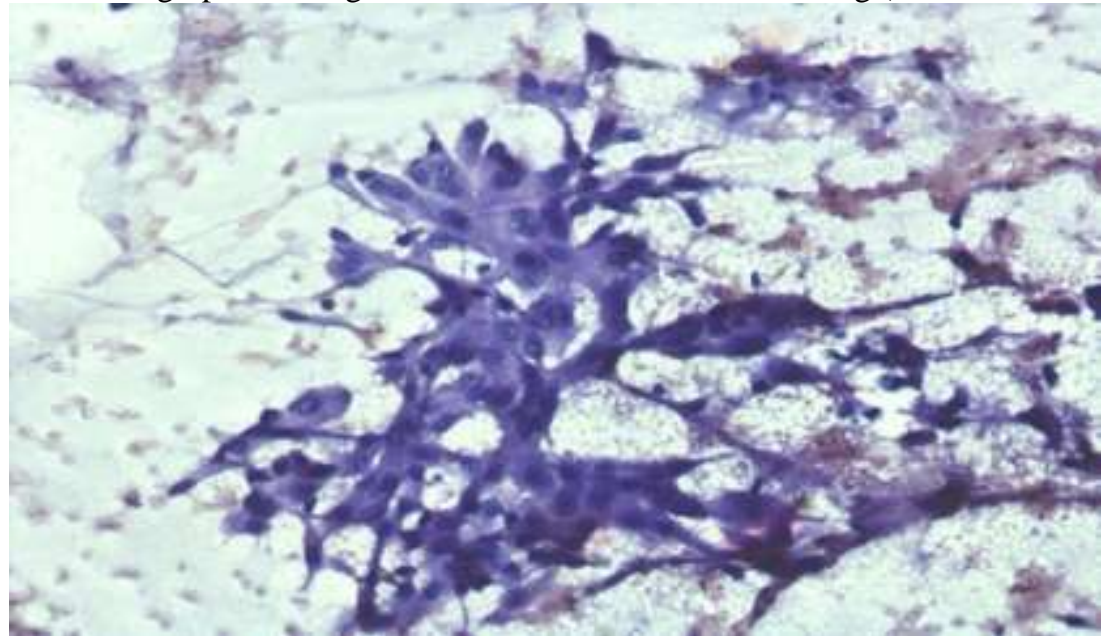

